\title{
Electron Cyclotron Resonance Ion Source plasma characterization by energy dispersive $\mathrm{X}$-ray imaging
}

\author{
R. Rácz ${ }^{1}$, D. Mascali ${ }^{2}$, S. Biri ${ }^{1}$, C. Caliri ${ }^{2}$, G. Castro $^{2}$, A. Galatà ${ }^{3}$, S. Gammino ${ }^{2}$, L. Neri ${ }^{2}$ J. Pálinkás ${ }^{1}$, F. P. \\ Romano $^{4}$, G. Torrisi ${ }^{2}$ \\ ${ }^{1}$ Institute for Nuclear Research, Hungarian Academy of Sciences (Atomki), Debrecen, Hungary \\ ${ }^{2}$ Istituto Nazionale di Fisica Nucleare - Laboratori Nazionali del Sud, Catania, Italy \\ ${ }^{3}$ Istituto Nazionale di Fisica Nucleare - Laboratori Nazionali di Legnaro, Legnaro, Italy \\ ${ }^{4}$ CNR - Istituto per I Beni Archeologici e Monumentali, Catania, Italy
}

Pinhole and CCD based quasi-optical X-ray imaging technique was applied to investigate the plasma of an Electron Cyclotron Resonance Ion Source. Spectrally integrated and energy resolved images were taken from an axial perspective. The comparison of integrated images taken of argon plasma highlights the structural changes affected by some ECRIS setting parameters, like strength of the axial magnetic confinement, RF frequency and microwave power. Photon counting analysis gives precise intensity distribution of the X-ray emitted by the argon plasma and by the plasma chamber walls. This advanced technique points out that the spatial positions of the electron losses are strongly determined by the kinetic energy of the electrons themselves to be lost and also shows evidences how strongly the plasma distribution is affected by slight changes in the RF frequency.

\section{INTRODUCTION}

Electron Cyclotron Resonance (ECR) Ion Sources (ECRIS) [1, 2] deliver variously charged ion beams for direct applications and, more frequently, highly charged ions worldwide for high energy accelerators. Ions in these sources are created and extracted from a plasma produced by ECR-discharge in a usually very strong B-minimum type magnetic trap. The parameters of the extracted ion beam are strongly determined by the features of the ECR plasma itself. Hence, the understanding of the plasma and its features is essential.

Many signs demonstrate that the ECR-discharge, i.e. the microwave-to-plasma interaction in the presence of strong external magnetic field, in a closed resonant cavity (ECRIS plasma chamber) is still not fully exploited $[3,4]$. One of the limiting factors in the complete understanding is the few types of diagnostics tools so far developed and installed especially because of the compactness of these devices. Any significant step forward in ECRIS physics and technology requires diagnostics tools of various types. Therefore since the development of the first ECR ion sources different plasma diagnostics methods were developed and adapted: microwave interferometry [5], X-ray spectroscopy [6, 7] (including precise energy and spatial resolution), visible light observations [8], and small-size electrostatic (Langmuir) probes [9, 10].

An ECR plasma emits radiation in a wide range of the electromagnetic spectrum. The physical properties of the radiation mirror back many features of the plasma that has originated such radiation. For the production of the highly charged ions, the X-ray domain may provide the most significant information on the plasma. Namely, the electrons having energy between about $3-10 \mathrm{keV}$ (so-called warm electrons) are very effective in the step-bystep ionization processes (taking place in ECRISs), are exciting atoms and ions, and thus produce both characteristic and Bremsstrahlung radiation.

In 2014 a series of X-ray measurements have been carried out at the Atomki ECR ion source [11]. The measurement was done in close collaboration between the Atomki ECRIS team (Debrecen, Hungary) and the ion source group of INFN-LNS (Catania, Italy).

The measurements allowed performing plasma X-ray measurements both in the high energy $(>30 \mathrm{keV})$ and medium energy (2-30 keV) domains, with the simultaneous extraction of the ion beam and the 2D X-ray imaging of the plasma by an X-ray pin-hole camera method. The technical details of applied tools and methods and also a detailed comparison with an earlier pioneering setup (carried out also at the Atomki ECRIS in 20022003 [7, 12]) have already been published [13]. During the measurements a huge number of data were recorded (X-ray single and multiple photos, energy spectra, etc.) and so far only a small part of the data was processed. The results of the analysis of the first part of the measured data were recently published $[14,15]$.

This present paper deals with detailed analysis of the second (larger) part of the measured data and thus considered as a logical continuation of the three previous articles [13-15]. Because both the experimental setup and the results of the first group of data were described in details in these papers, in the present paper here we give only their short summary. 


\section{THE EXPERIMENTAL SCENARIO}

The injection side of the cylindrical plasma chamber (ID=58 mm, L= $210 \mathrm{~mm}$ ) of the ECRIS was closed by a steel mesh to form a closed resonant cavity allowing meanwhile the penetration and detection of plasmaemitted X-ray photons. The overall sketch of the experimental setup showing all the detectors used during the experimental campaign are shown in the figure 1 in ref [13]. General layout of the plasma reactor (the Atomki ECRIS) can be find elsewhere [11]. We present only the specific part of the setup showing the layout of the imaging system in figure 1 . The magnetic trap consists of two identical room-temperature solenoid coils with $5 \mathrm{~cm}$ thick iron yoke and of a cylindrical shape (24 segments) permanent magnet hexapole. The radial pole field of the hexapole at the plasma chamber internal wall $(\mathrm{R}=29 \mathrm{~mm})$ is $1.2 \mathrm{~T}$ and the magnetic field strength along the plasma chamber axis as function of the coils currents is presented in fig1.

Two detectors were applied at the injection side, alternatively. The Silicon Drift Detector (SDD) was placed beyond a kapton foil for vacuum break, and collimated by a lead cylinder with a drilled hole. The CCD Xray camera made by $256 \times 1024$ pixels operational in the range $500 \mathrm{eV}-10 \mathrm{keV}$ was coupled to a lead pin-hole and placed along the axis, facing the chamber from the injection flange. Aluminium windows of variable thickness (range 1-6 $\mu \mathrm{m}$ ) were used to screen the CCD from the visible and UV light coming out from the plasma. The camera was used in two modes (written in details in the following section): a) integrated images were recorded (similarly to digital visible light cameras) to show the 2D spatial form of the plasma with some intensity details; b) spectrally-resolved images were recorded to get energy information on the impinging photons in each pixel or in any selected region of interest (ROI). In addition, High Purity Germanium (HPGe) detector was also placed on the axis, but at the other (extraction) side of the source beyond the 90-degree analysing magnet, closed to a quartz window. It was used for monitoring the X-rays emitted by the high energy electrons - from inside the plasma or when impinging on the chamber walls. The HPGe was collimated by lead blocks to detect the radiation passing through the extraction hole of the plasma chamber.

In the first series of measurements, the X-ray spectroscopy was performed by using the SDD and HPGe detectors characterizing the volumetric plasma emission. The direct main goal was to investigate the effect of the microwave frequency to the plasma and to the microwave-plasma interaction. SDD results revealed strong correlation between plasma and beam parameters (plasma parameters: X-ray counts, electron temperature and density; beam parameters: analysed beam intensity, average charge). The results revealed a surprisingly very good agreement between warm-electrons density fluctuations, output beam currents and the calculated electromagnetic modal density of the plasma chamber [14]. Several CCD pinhole-camera images were shown as example, pointing significant differences of the energy content and displacement of the plasma vs. microwave frequency.

During the measurements a wide range of external ion source setting parameters and technical solutions were tried and tested. The ECR ion source was optimized for producing $\mathrm{Ar}^{4+}$ ions, with magnetic trap strength able to guarantee ECR condition around $13 \mathrm{GHz}$. The RF power injected to the plasma chamber was kept constant (at $30 \mathrm{~W}$ ) in order to prevent high dead-times in the SDD detector and pixels-saturation in the CCD camera. The gas pressure measured at the injection side of the ion source was $\mathrm{P}_{\mathrm{inj}}=3.3 \times 10^{-6}$ mbar. X-ray spectra were taken at different pumping microwave frequencies between 12.80 and $13.40 \mathrm{GHz}$, with $40 \mathrm{MHz}$ steps (16 frequencies). Some of these frequencies proved to be better or worth than others, in terms of resulting higher or lower $\mathrm{Ar}^{4+}$ current. A limited number from them (so-called: "representatives") had to be selected for the time-consuming integrated ( 8 frequencies) and spectrally-resolved ( 3 frequencies) CCD recordings. The magnification of the pinhole system (developed by the position of the pinhole respect to the plasma and to the CCD chip) and the thickness of the Al-window (applied to cut the visible and UV part of the emitted spectrum) were optimised. The selection of these parameters for the X-ray imaging (frequencies, magnification, and thickness) and some effect of the external settings parameters of the ion source to the integrated plasma images were shown in a recent proceedings paper [15].

This work will present X-ray plasma images recorded by the CCD pinhole camera. The goal is twofold. (1) Through the detailed analysis of the integrated (not energy resolved) images to get information on the effect of several setting parameters (microwave frequency and power, magnetic field strength) on the structure of the plasma. (2) Through spectrally resolved images to get information on the source of the different X-ray components (characteristic X-ray photons emitted by plasma ions and by the atoms of the plasma chamber walls, Bremsstrahlung radiation created by the plasma electrons and by the lost and decelerated electrons in the plasma chamber wall) and on the spatial and line-integrated position of the plasma ions.

\section{SETUP FOR IMAGING WITH PINHOLE CAMERA}

Figure 1 shows the schematic drawing of the experimental setup used for X-ray plasma imaging. The arrangement allowed to take X-ray images, to extract ion beam and to record charge state distribution of the given plasma simultaneously. 
The area of the circular shape injection plate was divided into two regions by a ratio of 3:2. The smaller area was used for microwave and gas injection, while the larger part was closed by stainless steel mesh to keep a closed resonant cavity and to provide transparency for imaging. In addition, aluminum windows of variable thickness (range 1-6 $\mu \mathrm{m}$ ) were used to screen the CCD from the visible and UV light emitted also by the plasma. Lead shutter was applied to prevent the CCD while the ion source was tuned. Demagnification was necessary because the diameter of the plasma chamber is larger than the size of the CCD chip. The magnification (M) is the quotient of $d_{i}$ and $d_{o}$ (image and object distances). Three $M$ values were set and tried: 0.082, 0.124 and 0.158 . Also three different Al-windows were tested with thicknesses of 6, 1 and 3 micrometres (D).

Two different type of exposing methods were applied. In case of the spectrally integrated mode the photos are taken with several ten seconds exposure time. The only limitation for the exposure time is to avoid the blooming of the CCD (when a pixel in a CCD image sensor is overloaded). Individual pixels can be loaded by many X-ray photons, therefore the energy information of a given photon are lost. Photos were also taken in photon counting mode. In that case thousands of images are exposed with experimentally adjusted short (milliseconds) exposure time. Each pixel registered either 0 or $1 \mathrm{X}$-ray events. Individual pixel was used as a single photon detector to spectrally resolve the plasma image. Because of the strong inhomogeneity of the plasma, ROIs were selected and the exposure time was settled by the intensity of the given region. The intensity of the other parts of the images was left out of consideration.

According to the showed experimental setup X-ray images represented by Figure 2 were recorded. The red circles roughly show the contours of the farther (extraction side, small circle) and the closer (injection side, large circle) end-plates of the plasma chamber, respectively. The smallest white circle points the position of the extraction hole.

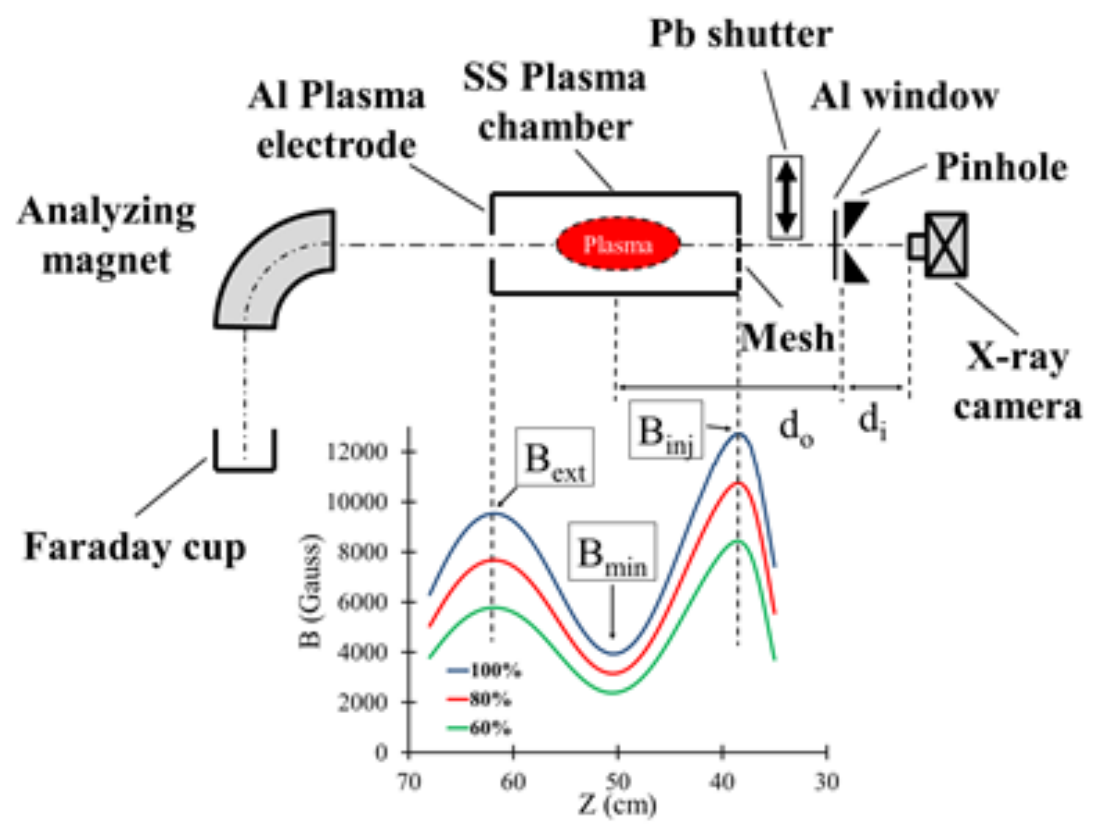

Figure 1. Schematic drawing of the experimental setup used for the X-ray plasma imaging and the magnetic field strength along the plasma chamber axis as function of the coils current.

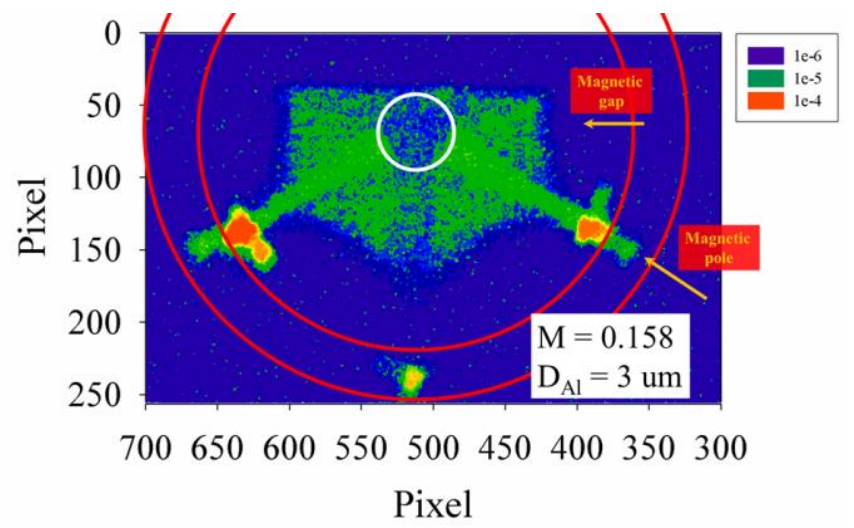

Figure 2. Typical integrated X-ray plasma image taken with 40 seconds acquisition time. The average magnification and the thickness of the aluminium window were 0.158 and $3 \mu \mathrm{m}$ respectively. Orientation of the 
hexapole magnet is showed by yellow arrows. The RF frequency was $12.84 \mathrm{GHz}$. Image was normalised to 1 (total number of counts in each picture $=1$ ) and mirrored by two axes to see exactly the original arrangement and plotted in logarithm colour scale with spectrum scheme.

\section{INTEGRATED IMAGES}

The effect of some external setting parameters of the ion source (microwave frequency, axial magnetic field, microwave power) to the spectrally integrated images were recorded and studied. Due to the long exposure time (15 - 40 seconds) the spectral information of a given pixel is lost, however the changes in the shape, structure and local energy contents of the plasma is clearly visible in the images presented by this chapter.

\subsection{Effect of the microwave frequency}

The effect of the externally coupled microwave frequency on the integrated images was studied. The frequency was varied between $12.84 \mathrm{GHz}$ and $13.16 \mathrm{GHz}$ with $80 \mathrm{MHz}$ steps. The microwave power coupled to the plasma chamber was 30 watt. The strength of the magnetic trap was maximal (100\% coils currents). Since this wide set of frequencies were applied only at the first series of the measurement, in the first half of this subsection the magnification and the window thickness are $M=0.082$ and $D=6$, respectively. The acquisition time was 15 seconds for each frame. Figure 3 shows the spectrally integrated plasma images taken at different frequencies.

It is evident from the images the density of the plasma along the plasma chamber axis is strongly affected by the RF frequency. ROI (white rectangle on the images) was selected corresponding to the extraction hole region to numerically represent the fluctuation of the X-ray counts. It was clear from the volumetric measurements that the intensity of X-ray photons emitted by the plasma is in strong correlation with the mean charge state $(\langle Q\rangle)$ of the extracted ion beam [14]. Figure 4 reveals the same dependence between $\langle Q\rangle$ (blue circle) and the total number of the counts of the given X-ray image (red triangles). The total counts measured at the near axis regions (in the selected ROI) respect to the total counts of the images (green squares in figure 4) are also following this fluctuation. One can conclude that the lower is the plasma density in the near axis region, the lower the $\langle\mathrm{Q}\rangle$. This highlights the effect of the density distribution of the plasma to the ionization efficiency.
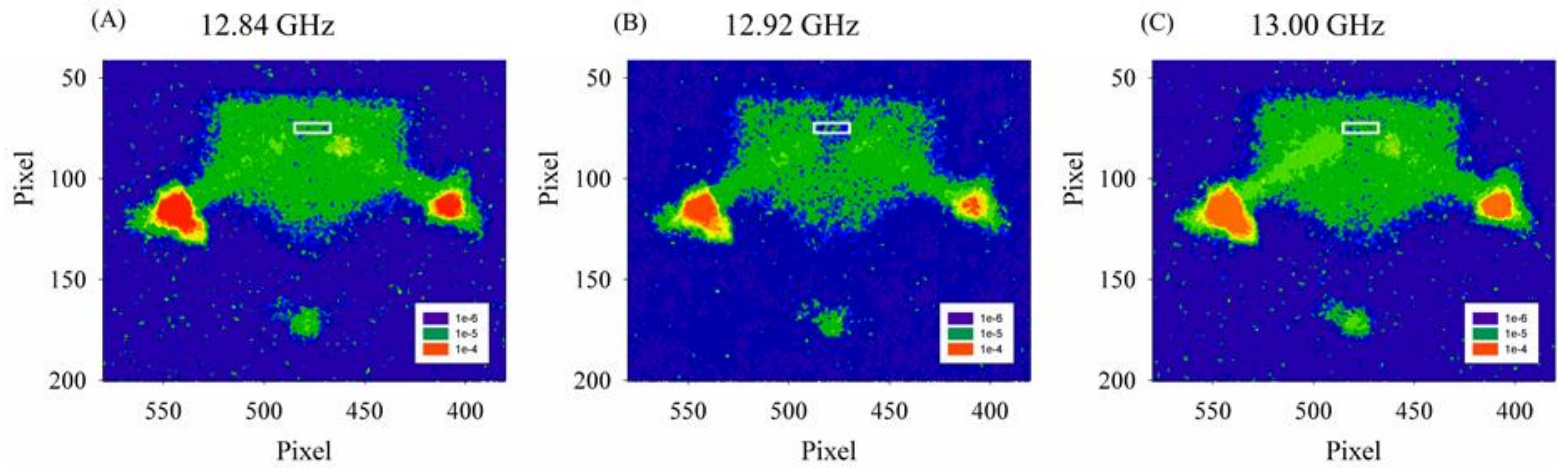

(D) $\quad 13.08 \mathrm{GHz}$

(E) $\quad 13.16 \mathrm{GHz}$
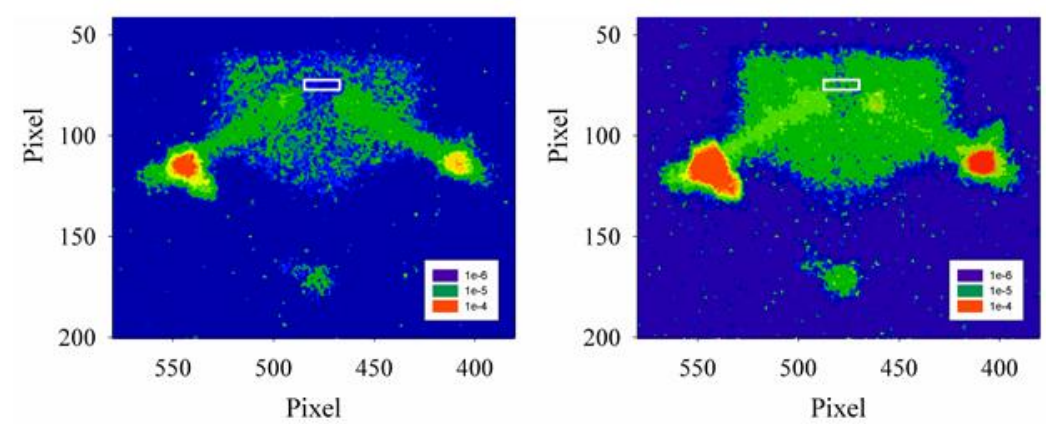

Figure 3. Spectrally integrated plasma images as function of the RF frequency. The images were normalised to 1 , mirrored by two axes and plotted in logarithm colour scale with spectrum scheme. 


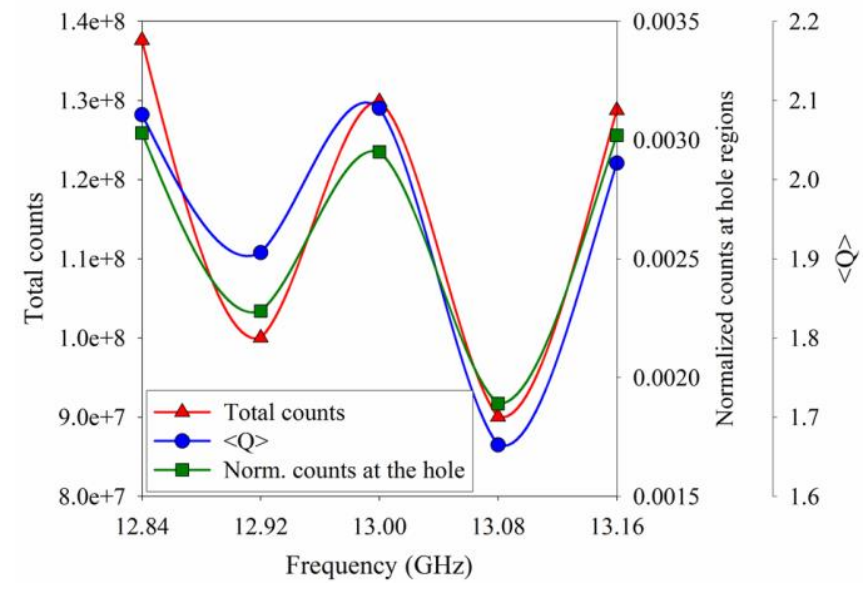

Figure 4. Mean charge of the extracted ion beam, total count of the X-ray frames, and the total counts measured at the near axis regions (extraction hole) respect to the total counts of the images as function of the RF frequency.

\subsection{Effects of the microwave power and of the strength of the axial magnetic field}

Effects of the microwave power and of the decrease of the axial confinement on the integrated images were recorded. The frequency was fixed at $12.84 \mathrm{GHz}$. X-ray images were exposed when the microwave power coupled to the plasma chamber was $20 \mathrm{~W}, 30 \mathrm{~W}$ and $40 \mathrm{~W}$. The strength of the magnetic trap was maximal (100 $\%$ coils currents). After that, the axial magnetic confinement provided by the solenoid coils were decreased while the injected microwave power was kept at fix $30 \mathrm{~W}$. Images were taken when the coils current was set to $100 \%$, $80 \%$ and $60 \%$ of the maximum output current of the power supply. By changing the currents on the coils all of $\mathrm{B}_{\mathrm{inj}}$ (injection side), $\mathrm{B}_{\min }$ (minimum value), $\mathrm{B}_{\text {ext }}$ (extraction side) values featuring the magnetic field configuration are changed. The changes are listed in table 1 . The average magnification and the window thickness were $\mathrm{M}=0.158$ and $\mathrm{D}=3$, respectively. The acquisition time was 40 seconds for each frame. The images were normalised to 1 and mirrored by two axes.

Table 1. Magnetic field strength along the plasma chamber axis as function of the applied coils current

\begin{tabular}{|c|c|c|c|}
\hline Coils current & $\mathrm{B}_{\text {inj }}(\mathrm{T})$ & $\mathrm{B}_{\min }(\mathrm{T})$ & $\mathrm{B}_{\text {ext }}(\mathrm{T})$ \\
\hline $100 \%$ & 1.26 & 0.39 & 0.95 \\
\hline $80 \%$ & 1.07 & 0.32 & 0.77 \\
\hline $60 \%$ & 0.84 & 0.24 & 0.58 \\
\hline
\end{tabular}

The spectrally integrated plasma images as function of the injected microwave power can be seen in figure 5. Horizontal profiles applying 10 pixel thick lines through the extraction hole are presented in figure 6 (B). It is clearly visible while the total count of the X-ray photos are increasing with microwave power (figure 6 (A)) the structure of the plasma in terms of the horizontal profiles remains the same.
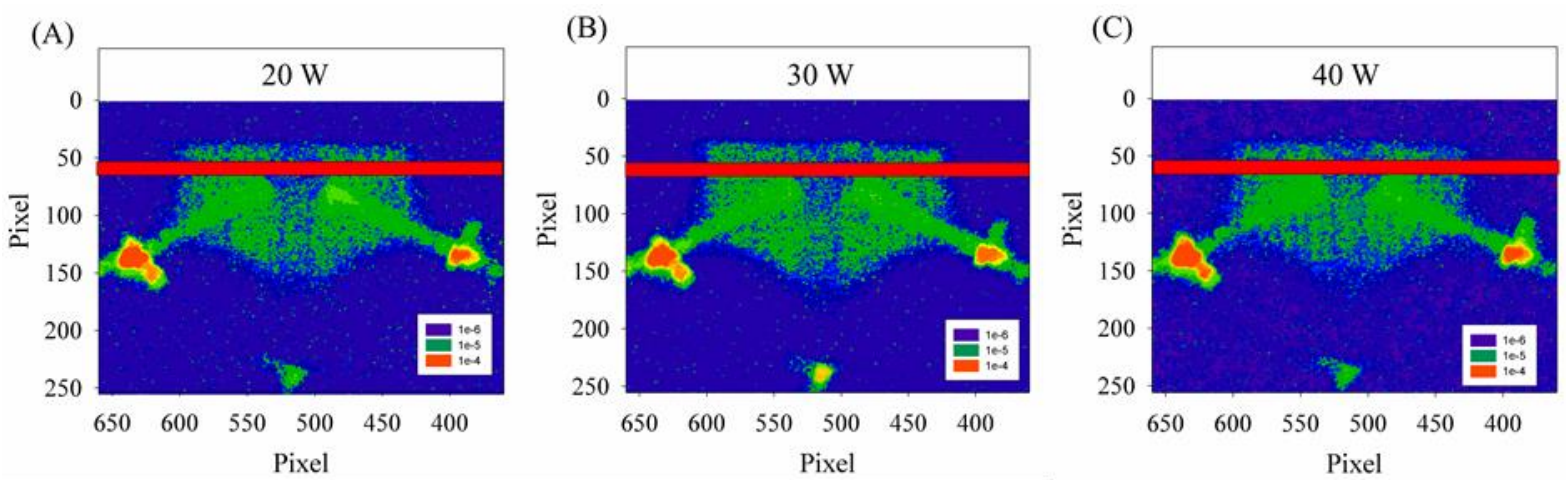

Figure 5. Spectrally integrated plasma images as function of the injected microwave power. The images were normalised to 1 , mirrored by two axes and plotted in logarithm colour scale with spectrum scheme. 


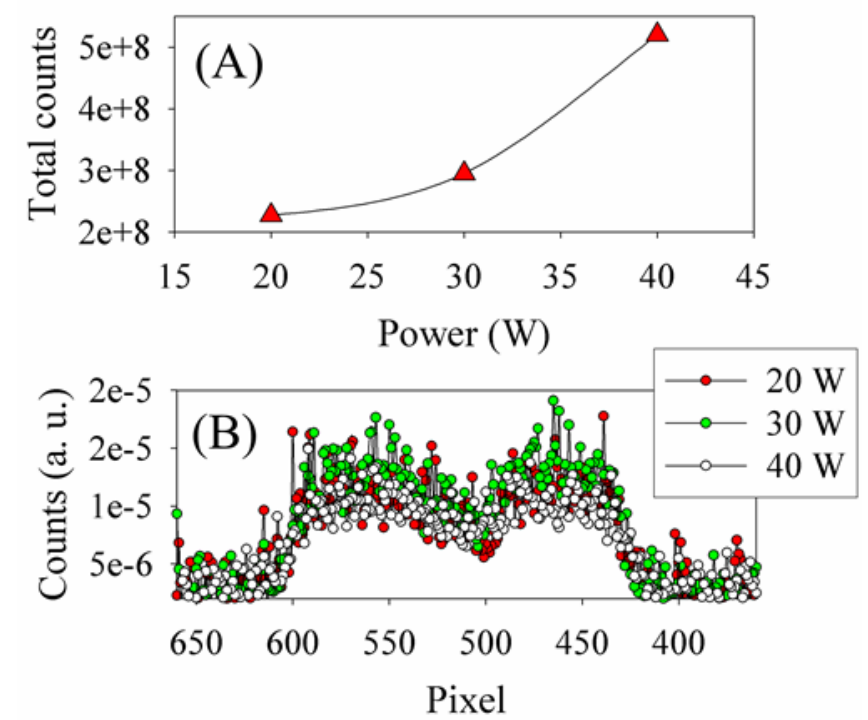

Figure 6. Total counts (A) and the horizontal density distribution of the counts (B) as function of the RF power.

Plasma images with the horizontal density distribution of the X-ray counts as function of the strength of the axial confinement are shown by figure 7. Similarly to the effect of the microwave power, the total counts of the images are increasing with the applied coils current, as shown by figure 7 (D). Furthermore, strong structural effects in the horizontal profiles are evident; the near axis region becomes emptier at each reduction step and the edge of the horizontal profiles (red circle in figure $7(\mathrm{E})$ ) is shifting toward the plasma chamber wall. This shift can be explained by the radial expansion of the resonant surface. The radial shift of the resonant layer affected by the decrease of the coils current was calculated in the real space. These shifts were transformed by the average magnification of the setup and by the pixel density of the CCD chip in order to estimate the shift on the CCD in pixel unit caused by the radial expansion of the resonant zone (blue square in figure $7(\mathrm{E})$ ). Good relative agreement was found between the estimated and the measured shifts. However, the measured shifts are systematically larger than the estimated one. This may come from the fact that by decreasing the axial confinement, the axial size of the ellipsoid-like resonant surface is increasing and it is getting closer to the pinhole. Therefore and because of the perspective imaging of the setup the measured shift must be larger than the one estimated by an average magnification value.
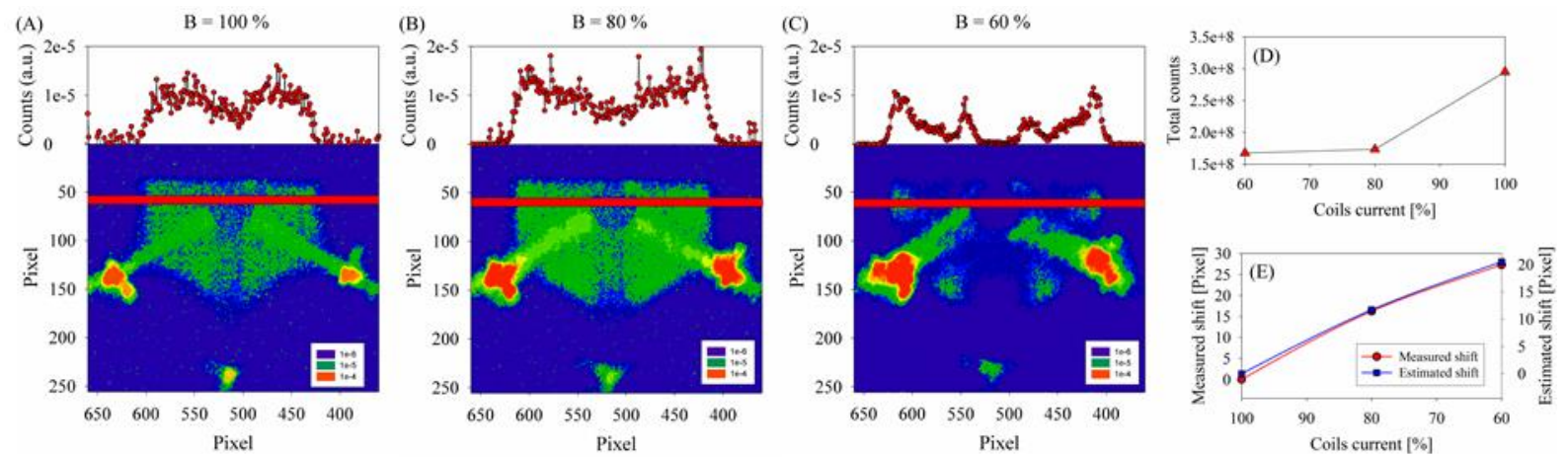

Figure 7. Spectrally integrated plasma images with the horizontal density distribution of the X-ray counts as function of the axial confinement $(A)-(C)$. Total counts of the given X-ray photos vs. axial B field (D). (E) shows the estimated and the measured shift of the edge of the horizontal density distribution profile affected by the degrease of the axial magnetic field strength. The images were normalised to 1, mirrored by two axes and plotted in logarithm colour scale with spectrum scheme.

\section{SPECTRALLY RESOLVED IMAGES}

The CCD camera was then used in single photon counting mode to obtain spatially and, at the same time, spectrally resolved images of the plasma. Thousands of frames were exposed with experimentally adjusted short (milliseconds) exposure time. During this time each pixel registered either 0 or $1 \mathrm{X}$-ray events at each frames. Thus each pixel operated as a single photon detector giving energy information (analogue digital unit (ADU)) at 
the location of any pixel. With this method, not only the 2D distribution of the photon sources could be detected, but also information on the local energy contents of the plasma, and thus about the energy of the electrons causing the radiation, can be obtained. The energy resolution is comparable to the traditional non-imaging solid state $\mathrm{X}$ ray detectors. The specific energy resolution depends on the readout speed and on the eventual "re-binning" of the CCD sensor. At the ones used for the present experiment ( $1 \mathrm{MHz}$, bin=1 pixel) it was around $225 \mathrm{eV}$ [16].

The huge amount of acquired raw data was handled by an on-purpose developed software based on MatLab. Every pixel of the images recorded in photon counting mode are carrying a complex information package: $\mathrm{x}$ and $\mathrm{y}$ position, intensity, energy information. In this four dimensions parameters space, part of the image or part of the spectra should be selected to visualize the obtained results. We have developed post process code in order to: (1)-(2) analyse the single frames and summarize them, (3) obtain spectral information on the selected part of the image and (4) spectrally filter the images.

(1) A single photon can excite a group of pixels, therefore the energy (ADU) information corresponding to the given photon can be assigned to several pixels. Hence we developed a code able to recognize the total amount of intensity that can be assigned to every single photon captured in each single frame. This algorithm was able to increase the spatial and energy resolution as described in [16].

(2) During the readout of the CCD camera the accumulated charges are transferred through the rows of the CCD from the top to the bottom, where the reading buffer is. During this transition, the CCD is still active so it is sensitive to the impinging photons. This cause that some photons are still detected but in a "wrong place", and as a consequence vertical "strips" in the images appear. Algorithm to eliminate this effect was written and applied. The algorithm is able to identify the amount of time needed for the transferring of information between the rows and the standard acquisition time of the CCD. From that analysis it is able to identify the amount of photons collected in a wrong position, starting from the spatial distribution of the photons collected during the standard acquisition time of the CCD: once identified a photon collected in the wrong position, the algorithm reallocate it in the right position. Figure 8 demonstrates the effectiveness of this evaluation by showing plasma images before and after the application of the algorithm.

(3) It is possible to select a dedicated part of the image (ROI) and to investigate the spectral composition of this area only.

(4) It is possible to select only the pixels of the frames which are loaded by the photons having energy corresponding to a well-defined energy range to show the intensity distribution of the pixels which are fulfilling the filtering requirements.

In the following subsections $(5.1,5.2 .5 .3)$ results of both evaluation methods will be shown. In case of the spectrally resolved images the average magnification and the thickness of the aluminium window were 0.158 and $3 \mu \mathrm{m}$ respectively.
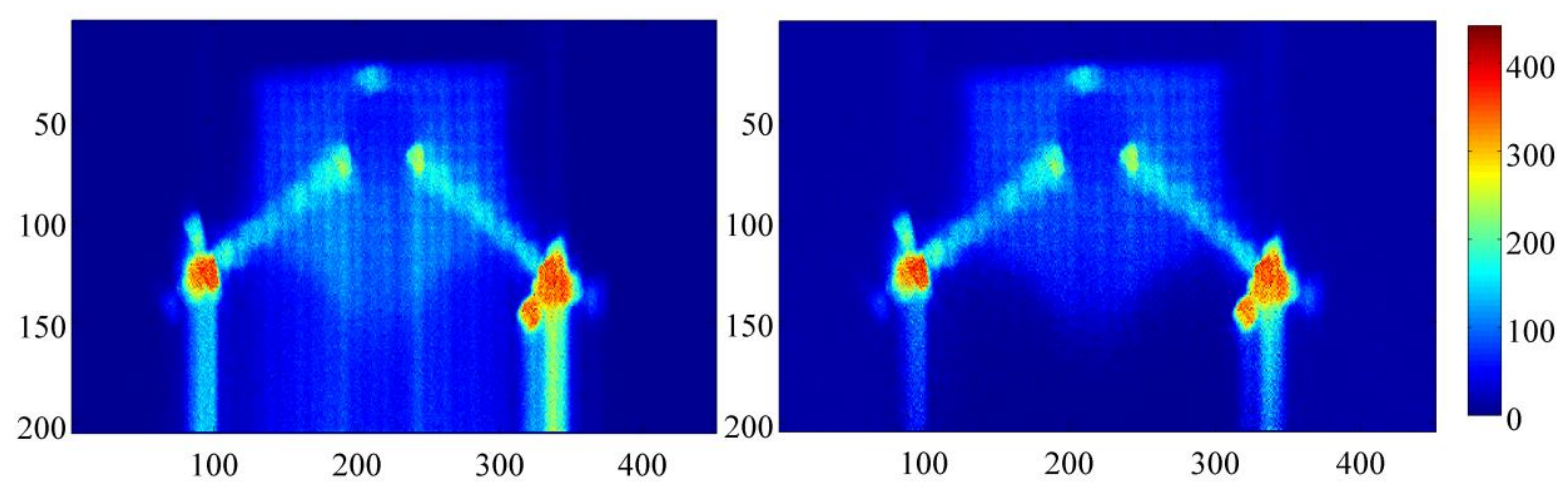

Figure 8. Comparative plot of photon counted images: before (left) and after (right) the application of the algorithm. The images were mirrored by two axes and plotted in linear colour scale with spectrum scheme.

\subsection{Spectra in ROIs}

Typical photon counted image of the plasma with the selected ROIs and the spectra of the full size image can be seen in figure 9 . 


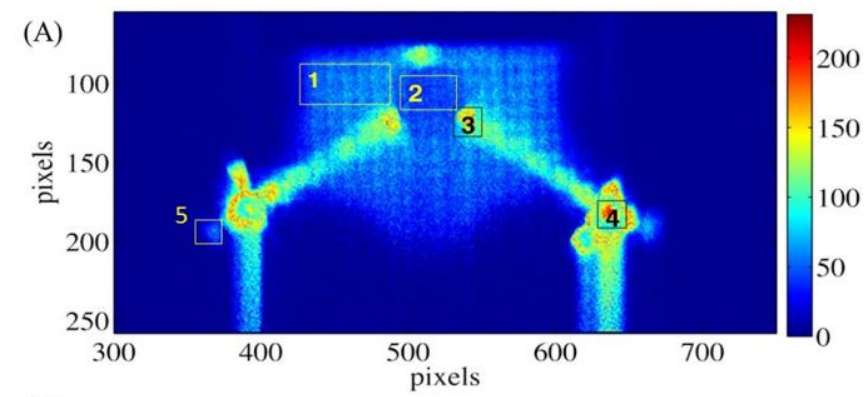

(B)

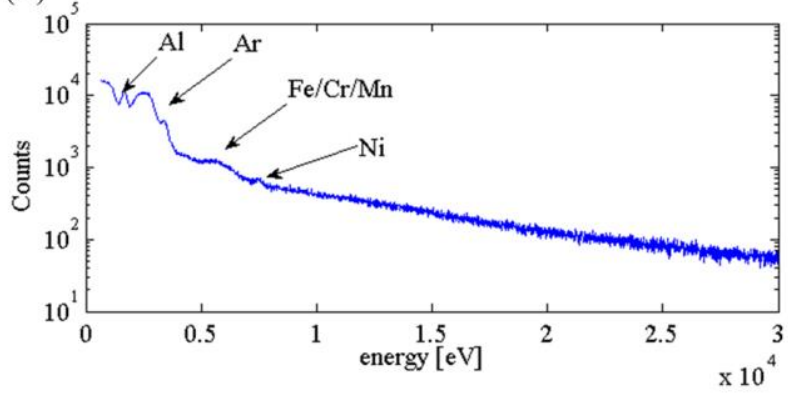

Figure 9. Superposition of the photon counted frames (A) and the corresponding X-ray spectra (B). Plasma parameters: Argon plasma, maximal magnetic field strength, $30 \mathrm{~W}$ microwave power, $12.84 \mathrm{GHz} \mathrm{RF}$ frequency.

The image was mirrored by two axes and plotted in linear colour scale with spectrum scheme. (A) shows the choice of the ROIs for the evaluation of the local spectral distribution of the emitted X-rays. The exposure time of one frame was $150 \mathrm{~ms}$. Spectra was normalized by the quantum efficiency function of the CCD camera.

ROIs were selected and named to show the local spectral composition of the following five regions, see figure 9(A): 1) plasma region, 2) extraction hole region, 3) intense region close to extraction hole, 4) extraction plate, 5) lateral wall of the plasma chamber. One can note that the intensity of the photon counted images (like figure 9 (A)) have a periodic grid-like modulation. It is caused by the mesh which was placed as injection endplate of the plasma chamber (see figure 1). The fact that the shadow of the grid elements is visible on the X-ray photos manifests the excellent spatial resolution of the imaging system. The spectrum showed by Figure 9 (B) includes the sum of the continuum and characteristic line emission components of plasma and plasma chamber walls. The continuum radiation originated from the Bremsstrahlung radiation generated by the deceleration of the energetic electrons colliding with heavy ions in the plasma and with the atoms of the plasma chamber walls. Characteristic peaks corresponding to the $\mathrm{K}$ lines of the argon, aluminum, iron / chromium / manganese and of nickel were identified.

Zoom of the plasma image at different ROIs with the corresponding spectra and the comparison of these spectrums for a given value of the pumping wave frequency $(12.84 \mathrm{GHz})$ can be seen in figure 10 and figure 11 , respectively.

The main characteristics of the full spectra (Fig. 9 (B).) are easy to be identified in the spectra of ROIs. Spectra measured at ROI-1 and ROI-2 are very similar to each other; beside the Bremsstrahlung radiation Ar-K $\alpha$ peak is dominant, representing the area of the Ar plasma. ROI-4 shows intense aluminum K $\alpha$ peak and Bremsstrahlung radiation: energetic electrons are lost on the Aluminum plasma electrode producing characteristic Al-K lines and continuum radiation. The intense spots close to the hole of the plasma electrode (ROI-3) shows the typical peaks measured at the plasma regions (ROI-1 and ROI-2) and at the extraction plate (ROI-4) as well. Because of the axial inspection the camera provides axially projected image of the plasma and the plasma chamber wall, emissions from Ar plasma and of the extraction plate are superimposed in ROI-3. The position of the ROI-5 corresponds to the lateral cylindrical wall of the plasma chamber as it was shown in figure 2 . The material of the plasma chamber of the Atomki ECRIS is KO36, AISI 321 stainless steel. It alloys nickel: 9 - $12 \%$, manganese: $\max 2 \%$, chromium $17-19 \%$. So the peaks obtained in the spectra of ROI-5 are reasonable showing the superposition of fluorescence lines coming from $\mathrm{Fe}, \mathrm{Mn}$ and $\mathrm{Cr}$ (they cannot be resolved due to the relatively low resolution of the CCD when operated at high sampling rate) and a well-defined line corresponding to Ni. The relatively pronounced $\mathrm{Ni}$ peak can be explained by the energy of the locally lost electrons. The height of a fluorescence peak, in fact, relies with the energy-dependent cross section of fluorescence lines excitation of the different elements. The spectrum in ROI-4 already shows that the spectrum of the electrons falling in the trap's loss cones have a rather peculiar trend with respect to the plasma ones. The low energy domains are in fact almost empty, while a broad "bump" extends toward high energies. Since the Ni lines are placed at higher energies with respect to the other elements of the SS, their excitation is relatively more probable, resulting in a higher intensity peak. While the energy distribution of $\mathrm{Ni}-\mathrm{K} \alpha$ is comparable with the $\mathrm{Al}-\mathrm{K} \alpha$ the Ar related peak $(\sim 3 \mathrm{keV})$ is significantly broader than the $\mathrm{Ni}$ one. Argon has not only $\mathrm{K} \alpha$, but also $\mathrm{K} \beta$ peak close to the energy of K $\alpha$. The 
sequence of energies is: Ar-K $\alpha 12.95770 \mathrm{keV}, \mathrm{K} \alpha 2=2.95563 \mathrm{Ar}-\mathrm{K} \beta=3.1905 \mathrm{keV}$. Those lines cannot be separated. Furthermore, the Ni peak is coming from the plasma chamber walls, and therefore this line emitted by SS crystal $(\mathrm{Ni}+)$, while the Ar peak corresponds to the multiple charged Ar ions. $\operatorname{Ar}^{\mathrm{q}^{+}}(\mathrm{q}=1-9)$ emit slightly energy-shifted X-ray lines whose energy lies within the energy resolution of the CDD: this also affects the broadening of the distribution. One can also identify Al peaks in ROI-5 spectra (despite the material of the lateral plasma chamber is stainless steel). We must note here, the Al plasma electrode has Al lateral walls longer than the widths of the plasma electrode (in order to fix it to the chamber). Therefore the last $2 \mathrm{~cm}$ of the SS plasma chamber cylinder (at the extraction side) is partly covered by Al. The spectrum produced by the radial loss lines "going toward" the extraction electrode (identified in ROI-5) correctly shows Al related peak. The peaks obtained at 4.6 $\mathrm{keV}$ in ROI-1, ROI-2, ROI-3 spectra cases (see Fig. 11.) cannot be identified with any of the elements in the plasma or composing the cavity walls. It probably is an artifact caused by an unknown process affecting the detector.
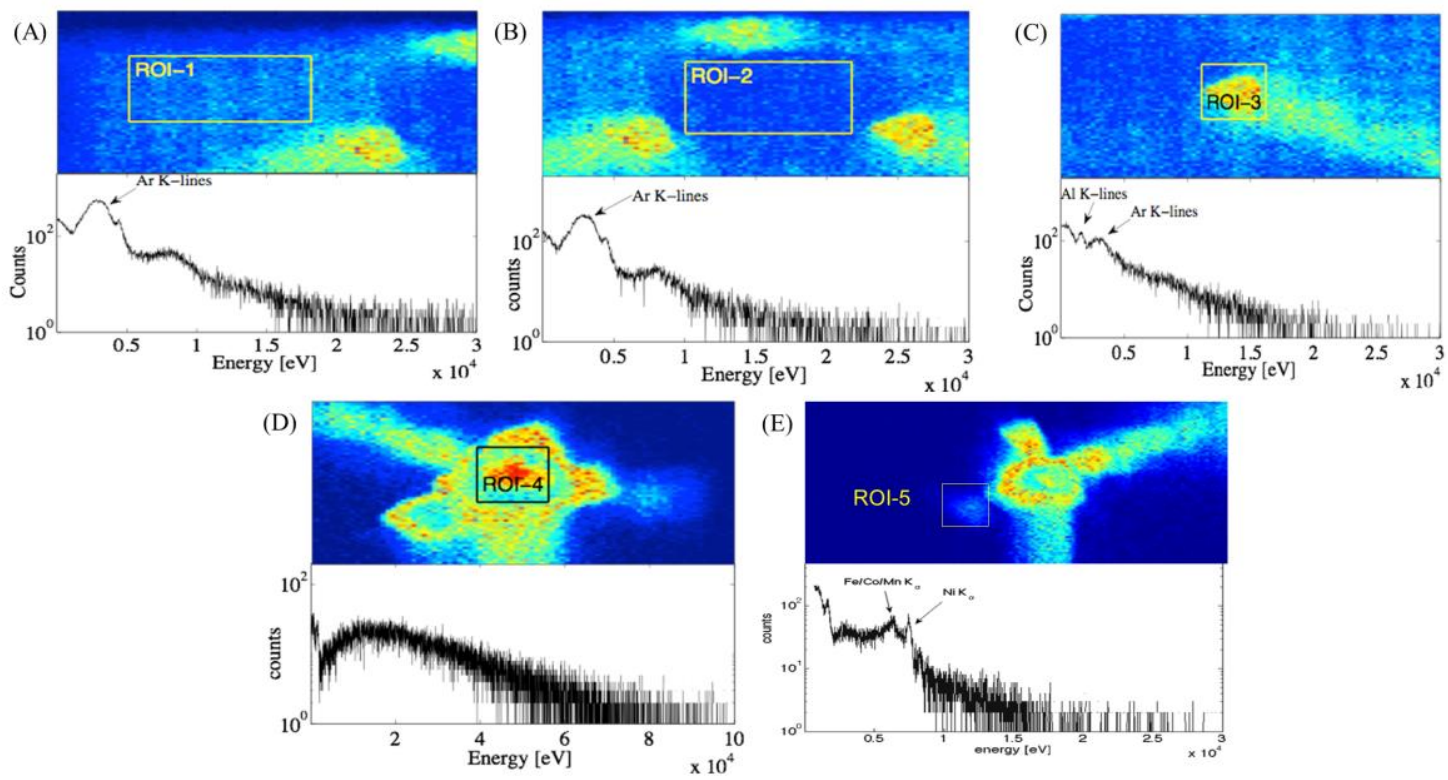

Figure 10. Zoom of the ROIs with the corresponding calibrated X-ray spectra.

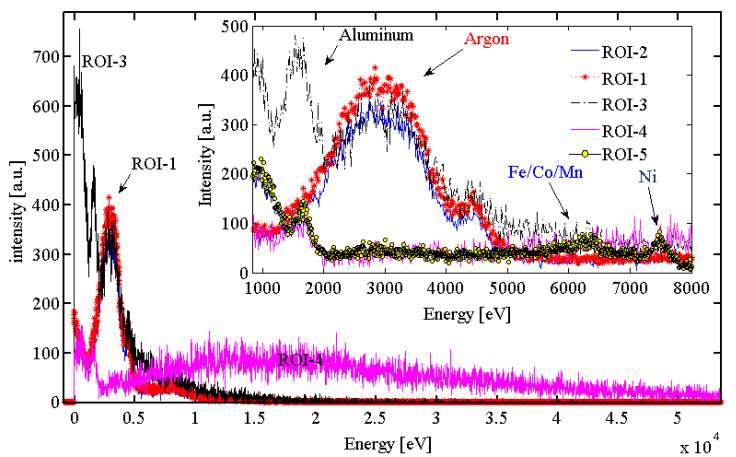

Figure 11. Comparison of the different spectra collected in the five selected ROIs.

\subsection{Spectrally filtered images}

Any X-ray plasma photos, like it is figure 9 (A), can be filtered by the energy of the X-ray photons. Consequently, the spectra like the one presented in figure 6 (B) were divided into three parts. Pixels of the frames which are loaded by the photons having energy corresponding to the following energy ranges were selected: $\mathrm{E}<2 \mathrm{keV}, 2$ $\mathrm{keV}<\mathrm{E}<4 \mathrm{keV}, \mathrm{E}>4 \mathrm{keV}$. The first group reveals to the "low" energy part of the spectrum, the second group was selected to represent the Ar-Ka characteristic line emission, while the third one is the "high" energy section. Pixels corresponding to these intervals were selected and plotted as function of the rf frequency in figure 12. By following the columns of this figure one can conclude that the spatial distribution of the Ar plasma is dense in off- 
pole position, while the low energy electrons are lost mainly axially, and furthermore, the losses of the high energy electrons are dominant along the radial direction.

\subsection{Effect of the RF frequency to the spectrally resolved images}

Effect of the microwave frequency to the spectrally resolved images was investigated. Spectrally filtered images and the spectral composition at different ROIs were analysed. $12.84 \mathrm{GHz}$ and $12.92 \mathrm{GHz}$ frequencies were selected for further studies as being the two frequencies producing the broadest fluctuation in both X-ray flux and output current, while $13.24 \mathrm{GHz}$ picked up to represents a significant frequency since the increase of output current does not correspond (as occurs for the other data) to a comparable increase of X-ray flux pointed by the volumetric measurements. Detailed description of the selection is written in [15]. Effect of the frequency is visualized in Figure 12 .
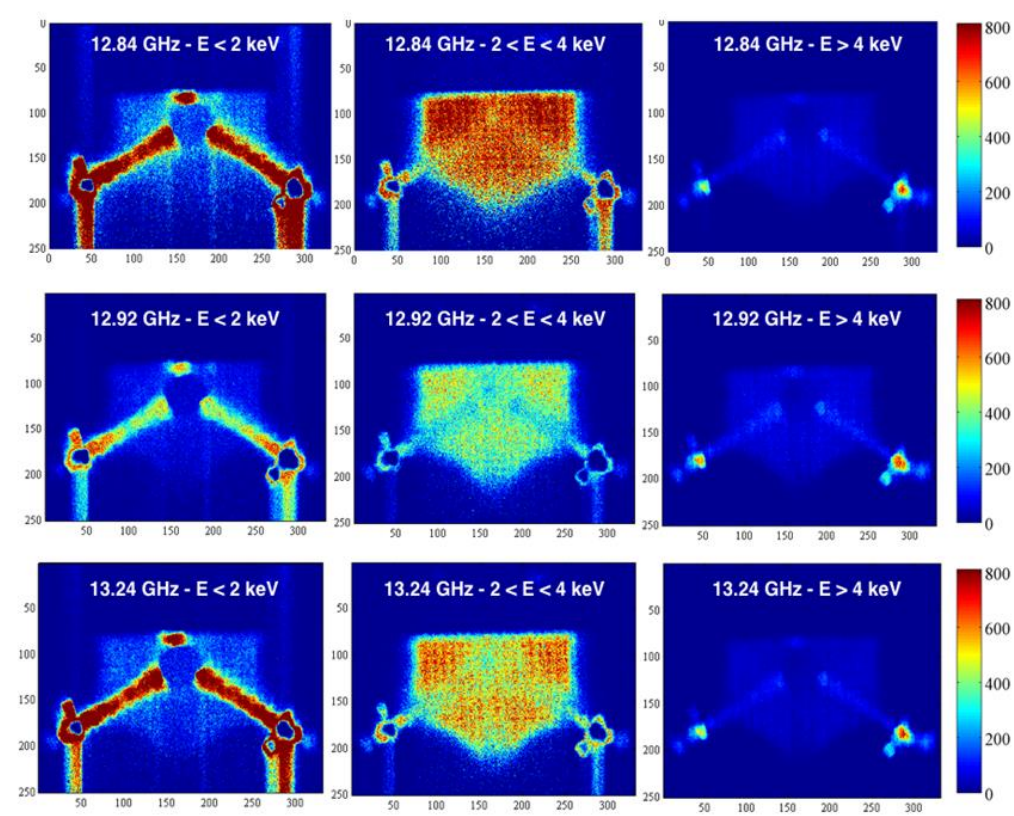

Figure $12.3 \times 3$ array of spectrally filtered images as function of the RF frequency (top to down) and as function of the energy defined in the previous subsection: low energy, $\mathrm{K} \alpha$, high energy (left to right). The images were normalized to the maximum intensity of the given frame, mirrored by two axes and plotted in linear colour scale with spectrum scheme.

Structural changes of the images are clear frequency by frequency. Plasma image filtered for Ar-K $\alpha$ emission is dense in off-axis and off-pole (magnetic gap) positions. It is proved not only by the images but also by the horizontal and azimuthal density profiles, see figure 13:

(1) The plasma is dense in the radial position of the resonant zone. The hollow type horizontal profile (depletion of axial density) is strongly affected by the RF frequency regardless from the absolute intensity of the image;

(2) Azimuthal profiles show a depletion at magnetic pole position. Since the magnetic field lines cross the lateral wall of the plasma chamber at magnetic pole position, the ions spend less time here than at gap positions, so the argon ion density is lower in the pole positions.

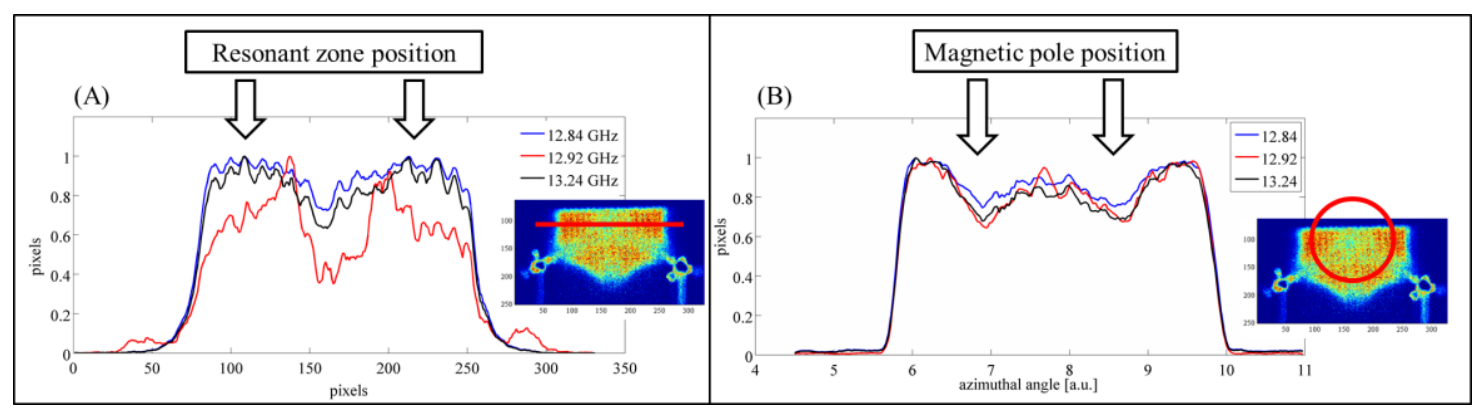

Figure 13. Horizontal and azimuthal profiles of the plasma images filtered for the Ar-Ka peak as function of the RF frequency. Profiles are plotted by summing up to 10 pixels vertically. Horizontal profiles were marked by arrows by the position of the resonant zone $(\mathrm{A})$. The arrows in $(\mathrm{B})$ indicate the magnetic pole position in the 
azimuthal profiles. The periodic fluctuations so clearly visible in the horizontal profiles are caused by the gridelements (used to close the resonant cavity at the injection side of the plasma chamber). Profiles were normalized to the maximum intensity.

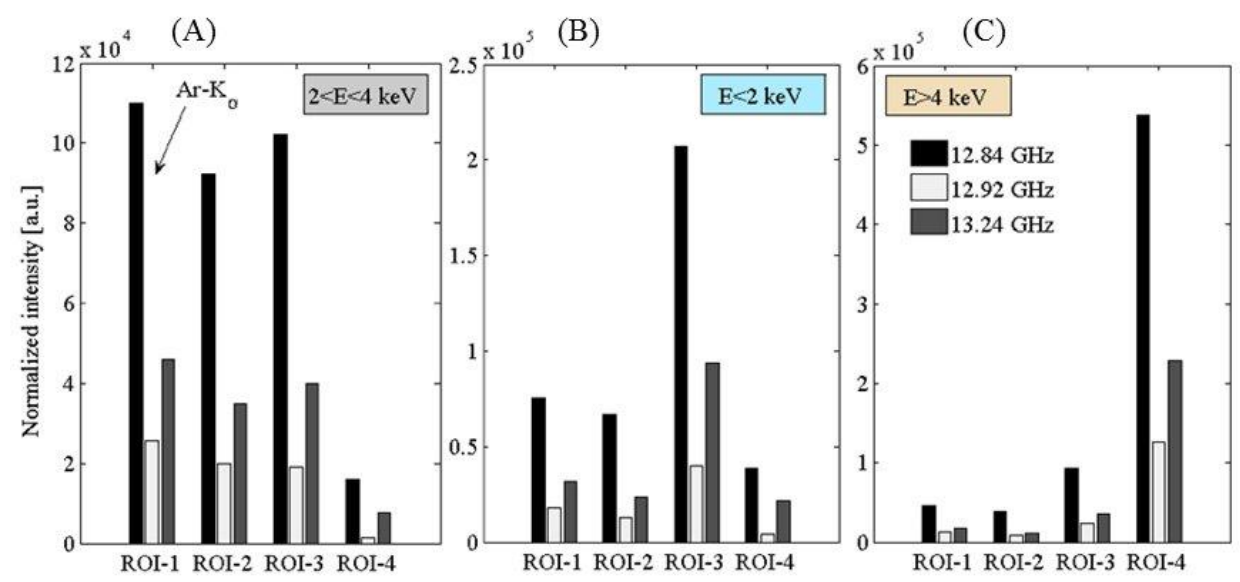

Figure 14. Total intensity (normalized with respect to the ROIs surface in number of pixels and to the exposure time) for each selected pumping wave frequency, and versus the selected ROIs. The plots refer to the range of

Ar-Ka lines (A), to the low energy electrons (B) and to the high energy electrons respectively (C).

The spectral composition of the plasma image at the ROIs was compared for the three selected frequency (figure 14).The spectrums were divided into three groups by the same energy ranges as described in the previous section. The first plot figure 14 (A) roughly gives an idea of Ar concentration in the different parts of the plasma, figure 14 (B) and figure 14 (C) refers to the low and high energy electrons respectively. This complex figure helps to conclude:

(1) High energy electrons are dominant in the radial positions close to the lateral chamber wall (in ROI-4), azimuthally at the magnetic poles of the hexapole (see Fig. 9 and Fig. 14 (C)). This trend is independent from the RF frequency.

(2) Losses of the low energy electrons (Fig. 14(B)) are dominant at close to the axis of the plasma chamber (ROI3). The losses are more than two times higher at ROI-3 respect to the other part of the plasma chamber (ROI1, ROI-2, ROI-4). This trend is independent from the RF frequency.

(3) Argon ions more concentrate at off-axis position since the normalized intensity of ROI-1 is higher than in ROI-2 at all the frequency cases.

(4) Concentration of Ar ions (Fig. 14(A)) in ROI-1 and ROI-2 demonstrate that the $13.24 \mathrm{GHz}$ trend is very similar to the 12.84 one (despite the intensity at each ROI is almost three times lower): in ROI-3 there is an increase of the intensity with respect to ROI-2. However this trend is conversely obtained at the case of 12.92 GHz. For $12.92 \mathrm{GHz}$ the emission is decreasing monotonically from ROI-1 to ROI-4. This can explain the fact that at certain frequencies there is a non-perfect correlation between the volumetric emission and the trend of the $\mathrm{Ar}^{4+}$ current, as evidenced in our previous paper [14].

\section{DISCUSSION AND COMPARISON WITH SIMULATIONS}

The powerful diagnostic method adopted for obtaining structural information of the plasma is particularly useful for investigating the deep plasma dynamics which are at the basis of the ion beam formation in ECRIS. The experimental data demonstrate that:

- The plasma shape (in the examined electron energy ranges) is intrinsically "hollow", with most of the electrons concentrated in the near resonance region, as well as plasma ions, which additionally lie in the off-pole regions, leaving a "hole" in the near axis zone;

- The "hollowness" of the plasma structure can be more or less emphasized by changing the plasma generation parameters (especially, magnetic field and RF frequency). However, despite the effect of the magnetic field was expected, a so deep influence of the pumping wave frequency on the structural properties of the plasma is something new. It was indirectly observed at GSI-Darmstadt [17], predicted by theoretical papers [18], but never observed so clearly (and directly into the plasma chamber) up to now.

The two points above open wide two interesting issues. The first is that, probably, the actual mechanism of plasma heating in minimum-B structures is not the one maximizing the efficiency of ion beam generation. Ions, in facts, are extracted electrostatically from an extraction hole placed on one of the two cylindrical plasma chamber endplates. The hole is collinear with the chamber. Only few magnetic flux tubes depart from the high density plasma contained inside the resonance zone and reach the extraction hole. Due to the moderate curvature of the 
magnetic field lines in the near axis region, ions are taken from the near axis regions as well, hence delivered to the extraction hole. Therefore, hollow plasmas will produce lower ion beam currents, and, sometimes, hollow beams as well [17].

The results illustrated along the paper, therefore, suggest revising the microwave power deposition mechanism, trying to maximize the absorption of the RF power into the plasma core [19].

The confirmation that the characteristic shape of the plasma obtained by the imaging technique is intrinsically related to the plasma formation mechanism in B-minimum structures comes also from numerical simulations.

We performed a series of simulations based on the quasi-self consistent approach developed in [20] and [21]. The code conjugates FEM solutions of the Maxwell equations [20] including the resonator (metallic plasma chamber) effects and the "cold" dielectric tensor of the plasma, with a "particle mover", i.e. a kinetic code developed in MATLAB for solving the single particle equation of motion of thousands of electrons according to the Boris method, and in the relativistic regime. Assuming a quasi-stationary equilibrium, particles can be followed until they "die" (i.e., they imping on the chamber walls), while their trajectories are stored in a 3D mesh which finally produce a charge density distribution, to be used at the next steps for computing electromagnetic field distribution. After the second step of interaction between the electromagnetic solver and the particle mover, effects of the particles on the field come out, and vice-versa.

For the present setup, we stop the interplay at the step number 2 (i.e. we evaluated two times the e.m. influence on the particles and vice-versa). We selected the two most significant frequencies explored during the experiment, i.e. 12.84 and $12.92 \mathrm{GHz}$. According to the "classical" scaling laws so-far adopted in ECRIS field, such a small difference in the exciting frequency should not affect at all plasma dynamics in terms of both density and temperature. The experimental data, conversely, demonstrate the influence is huge. This is already a proof for arguing that something linked to the frequency itself - i.e. the complex coupling of the wave with the plasma-filled resonator - is the key for explaining the experimental results. In the simulations we selected only one magnetic configuration (100\% current level in the coils) and fixed input microwave power, i.e. $30 \mathrm{~W}$ like in the reality, reproducing the same cavity size, the same magnetic field profile (including the axial simple mirror configuration and the radial hexapolar field). Results are shown in the sequence of figures 15-17. It is worth clarifying that simulated 2D maps of density were taken for only some specific energy intervals. No local EEDF mapping is possible at the present time. We selected some significative energy ranges as it follows: $\mathrm{E}<2 \mathrm{keV}$ (they are hereinafter called "cold electrons"); $2<\mathrm{E}<30 \mathrm{keV}$ (hereinafter called "warm electrons"); E $>30 \mathrm{keV}$ (hereinafter called "hot electrons"). In particular, figure 15 shows the 3D structure of the plasmas as it comes out from the simulations at the step no. 2. A "cold" plasma core $(\mathrm{E}<2 \mathrm{keV})$ is populated by low energy electrons, while the warmer particles $(2<\mathrm{E}<30 \mathrm{keV})$ form an envelope in the "equatorial" region. The figure shows that the most part of electrons that are lost are actually cold, and flow away from the plasma along the chamber axis (this is consistent with well-known effects in ECRIS like the bias-disk mechanism [22]). The warm electrons, instead, concentrate in the near resonance region, forming a hollow structure. In addition, the comparative inspection of the two figures confirm that at $12.84 \mathrm{GHz}$ a larger volume of warm particles is produced if compared to $12.92 \mathrm{GHz}$, consistently with the experimental observations about X-rays and produced beam.

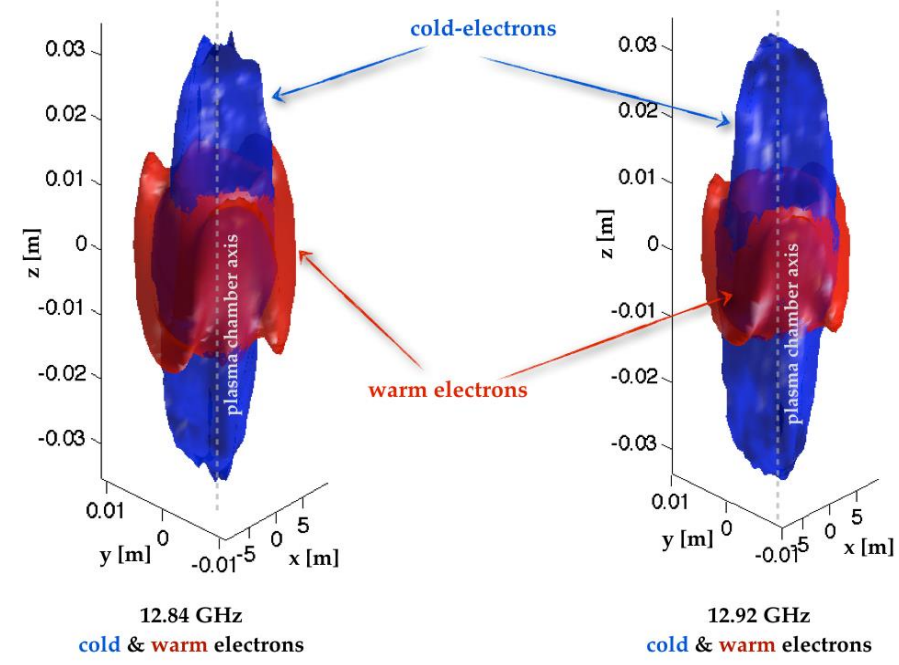

Figure 15. 3D structure of the plasma as it comes out from simulations. The main feature is that a "cold" plasma core is surrounded by a "warm" envelope that is bigger in volume when the plasma is excited by the modes at $12.84 \mathrm{GHz}$ instead than at $12.92 \mathrm{GHz}$. 
(A)

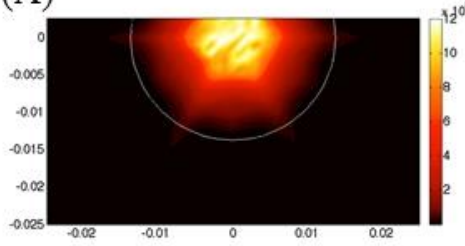

Cold Electrons $\mathrm{E}<1 \mathrm{keV}$

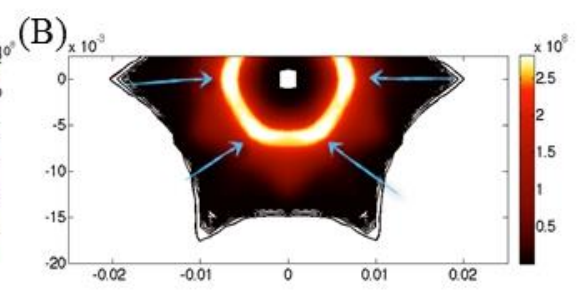

Warm electrons

$2 \mathrm{keV}<\mathrm{E}<30 \mathrm{keV}$

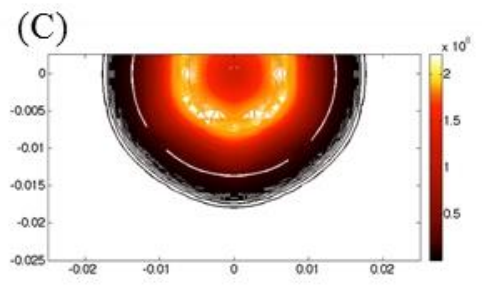

Hot electrons $\mathrm{E}>30 \mathrm{keV}$

Figure 16. Sequence of 2D images representing the perspective (transversal) distribution of the plasma electrons at different energy domains, for $\mathrm{f}=12.84 \mathrm{GHz}$. The same "cut" as in the experimental case was adopted for a direct comparison, done in the subsequent figure. Scales on the axes are in meters. The pseudo-color linear scales are given in arbitrary units with "hot" (black means low intensity, white means high intensity) scheme.

The situation is even clearer if one looks to the sequence of figures 16: (A) cold electrons are concentrated in the near axis region; (B) the ones with energy between 2 and $30 \mathrm{keV}$ (responsible of stepwise ionisation) are displaced within the resonance volume, not far from its surface, leaving a deep empty region in the near axis zone; (C) superhot particles, although preserving a hollow-shape, distribute in a smoother way. It is particularly interesting to note that the warm electrons also concentrate in the off-pole regions, as highlighted by the arrows.

A direct comparison between simulated and experimental data is presented in figure 17 . There the transversal distribution of the electrons in the energy range $2-30 \mathrm{keV}$ is compared with the filtered X-ray images corresponding to the $\mathrm{K} \alpha$ lines of the Argon. As mentioned above, the brighter emission at $12.84 \mathrm{GHz}$ corresponded to a larger output beam current. The simulations are consistent with such experimental evidence, since also the number of warm particles is larger at $12.84 \mathrm{GHz}$ rather than at $12.92 \mathrm{GHz}$, and the area populated from these particles is broader at $12.84 \mathrm{GHz}$.
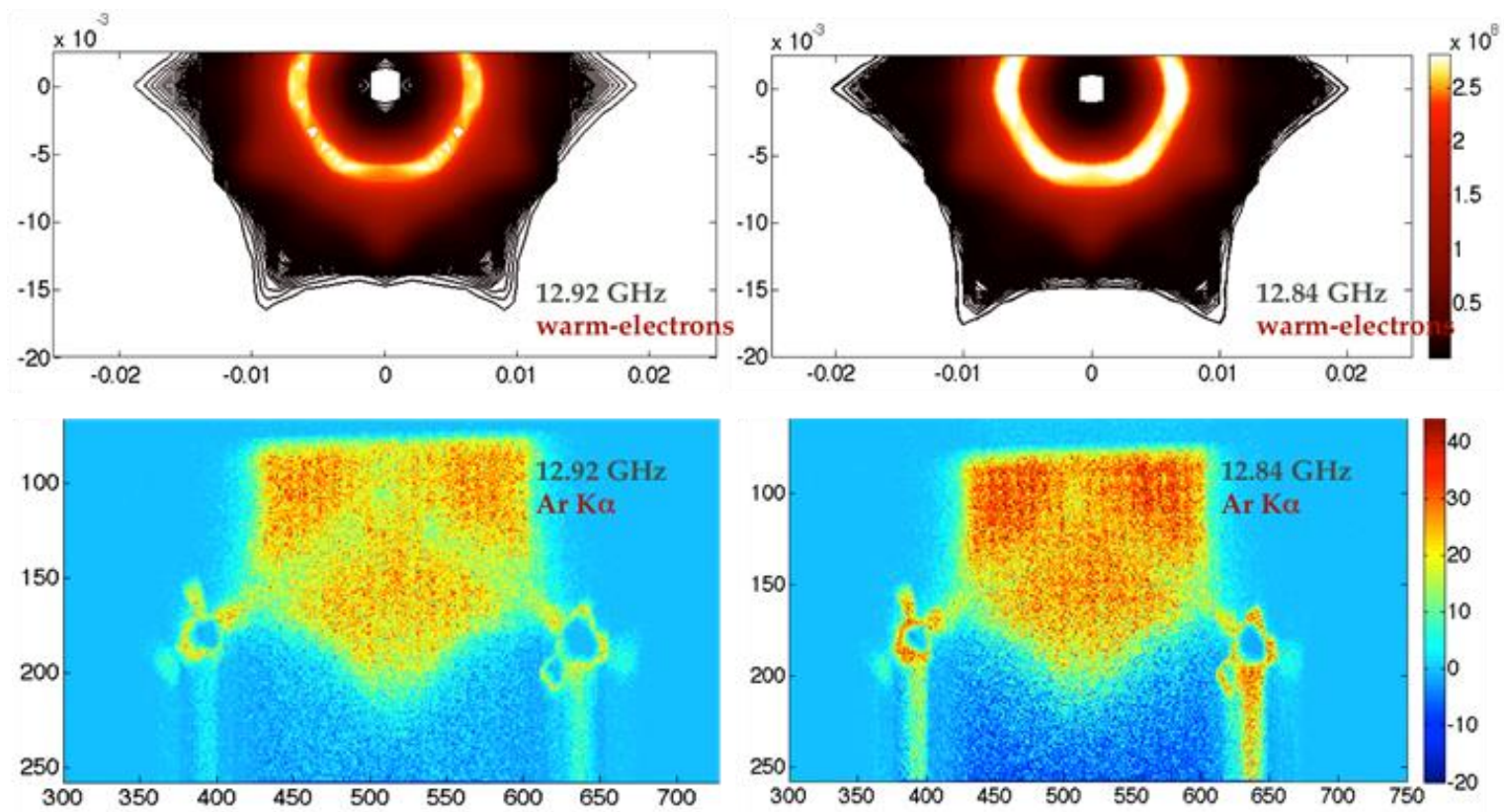

Figure 17. Direct comparison between simulations (up) and experimental data (down), for the two selected operative frequencies. The distribution of warm electrons is directly compared to the Ar ions distribution, since the formers are the responsible of the excitation of the latters. The images were normalized to the maximum intensity of the given frame, mirrored by two axes and plotted in linear colour scale with spectrum scheme.

The comparison must be then taken as qualitative: we would like to show that the electrons which are responsible of the stepwise ionization are displaced in a way that is compatible with the displacement of the ions into the plasma, as observed in the experiment. In a future paper, we will also compute local EEDF, including fluorescence generation cross-section. The pictures should get closer and closer to the reality. 


\section{SUMMARY}

A powerful diagnostic method was presented and used to analyse the axially projected spectrally and spatially resolved X-ray flux emitted by the ECR plasma and plasma chamber complex. Integrated and spectrally resolved images were analysed.

Effects of the fine frequency tuning, microwave power, axial magnetic confinement to the spectrally integrated images were presented. Strong effect of the RF frequency on the plasma images especially in the near axis region was demonstrated and numerically correlated with the $\langle\mathrm{Q}\rangle$ of the extracted ion beam. Strong effect of the axial magnetic confinement on the radial dimensions of the plasma was also highlighted: the plasma is expanding and is shifting toward the plasma chamber wall meanwhile the plasma images in the near axis region becomes emptier at each reduction step. The radial expansion of the plasma was explained by the radial expand of the resonant zone. No remarkable effect was observed on the plasma structure as a function of the externally coupled microwave power.

Analysis methods to handle the spectrally resolved images were presented. By several ways general features of the plasma images were shown and they can be summarized as follows:

- The multipole trapped ECR plasma is dense in the radial position of the resonant zone. The hollow type horizontal profile (depletion of axial density) is strongly affected by the RF frequency.

- The azimuthal profiles have depletion at magnetic pole positions, while the high-energy electron losses are dominating in these azimuthal positions.

- $\quad$ By analyzing the spectrally filtered counts at different ROIs trend obtained by the volumetric measurement as a function of the RF frequency was explained.

In the next future we are planning to combine the presented diagnostics tool with the simultaneous use of Langmuir probes and to apply it for the investigation of two frequency heating phenomena.

\section{ACKNOWLEDGEMENT}

"This project has received funding from the European Union's Horizon 2020 research and innovation program under grant agreement No 654002 (ENSAR2-MIDAS)". The experimental setup development has been also supported by the INFN V Nat. Comm. under the grants UTOPIA and RDH.

\section{REFERENCES}

[1] Proc. of the 16th Int. Conference on Ion Sources (New York, USA, 23-28 August 2015), Rev. Sci. Instrum. 87 (2016)

[2] Proc. of the 22nd Int. Workshop on ECRIS (Busan, Korea 28 August - 1 September 2016) www.jacow.org (publishing in progress)

[3] O. Tarvainen, T. Kalvas, H. Koivisto, J. Komppula, R. Kronholm, J. Laulainen, I. Izotov, D. Mansfeld, V. Skalyga, V. Toivanen and G. Machicoane, Limitation of the ECRIS performance by kinetic plasma instabilities, Rev. Sci. Instrum. 87 (2016) 02A703

[4] L. Celona, S. Gammino, G. Ciavola, F. Maimone and D. Mascali, Microwave to plasma coupling in electron cyclotron resonance and microwave ion sources, Rev, Sci. Instrum 81 (2010) 02A333

[5] D. Mascali, G. Torrisi, O. Leonardi, G. Sorbello, G. Castro, L. Celona, R. Miracoli, R. Agnello and S. Gammino, The first measurement of plasma density in an ECRIS-like device by means of a frequency-sweep microwave interferometer, Rev. Sci. Instrum. 87 (2016) 095109

[6] D. Mascali, L. Celona, F. Maimone, J. Maeder, G. Castro, F. P. Romano, A. Musumarra, C. Altana, C. Caliri, G. Torrisi, L. Neri, S. Gammino, K. Tinschert, K. P. Spaedtke, J. Rossbach, R. Lang and G. Ciavola, X-ray spectroscopy of warm and hot electron components in the CAPRICE source plasma at EIS testbench at GSI, Rev. Sci. Instrum. 85 (2014) 02A956

[7] S. Biri, A. Valek, T. Suta, E. Takács, Cs. Szabó, L. T. Hudson, B. Radics, J. Imrek, B. Juhász and J. Pálinkás, Imaging of ECR plasmas with a pinhole x-ray camera, Rev. Sci. Instrum. 75 (2004) 1420

[8] R. Rácz, S. Biri, J. Pálinkás, ECR plasma photographs as a plasma diagnostic, Plasma Sources Sci. Technol. 20 (2011) 025002

[9] J. L. Jauberteau, I. Jauberteau, O. D. Cortázar and A. Megía-Macías, Time evolution of the electron energy distribution function in pulsed microwave magnetoplasma in H2, Phys. Plasmas 23 (2016) 033513

[10] L. Kenéz, S. Biri, J. Karácsony, A. Valek, T. Nakagawa, K. E. Stiebing and V. Mironov, Diagnostic research of plasmas generated by a 14.5 GHz ECR ion source using Langmuir-probe, Rev. Sci. Instrum. 73 (2002) 617

[11] S. Biri, R. Rácz and J. Pálinkás, Status and special features of the Atomki ECR ion source, Rev. Sci. Instrum. 83 (2012) 02A341

[12] E. Takács, B. Radics, C. I. Szabó, S. Biri, L. T. Hudson, J. Imrek, B. Juhász, T. Suta, A. Valek, and J. Pálinkás, Spatially resolved X-ray spectroscopy of an ECR plasma - indication for evaporative cooling, Nucl. Instr. and Meth. in Phys. Res. B 235 (2005) 120 
[13] R. Rácz, S. Biri, J. Pálinkás, D. Mascali, G. Castro, C. Caliri, F. P. Romano and S. Gammino, X-ray pinhole camera setups used in the Atomki ECR Laboratory for plasma diagnostics, Rev. Sci. Instrum. 87 (2016) 02A741

[14] D. Mascali, G. Castro, S. Biri, R. Rácz, J. Pálinkás, C. Caliri, L. Celona, L. Neri, F. P. Romano, G. Torrisi and S. Gammino, Electron cyclotron resonance ion source plasma characterization by X-ray spectroscopy and $X$ ray imaging, Rev. Sci. Instrum. 87 (2016) 02A510

[15] R. Rácz, S. Biri, J. Pálinkás, D. Mascali, G. Castro, C. Caliri, L. Neri, F. P.Romano, S. Gammino, Structural Information on the ECR Plasma by X-Ray Imaging, 2017 Proc. 22nd Int. Workshop on ECRIS (Busan, Korea 28 August - 1 September 2016) ) www.jacow.org (publishing in progress)

[16] F. P. Romano, C. Caliri, L. Cosentino, S. Gammino, L. Giuntini, D. Mascali, L. Neri, L. Pappalardo, F. Rizzo and F. Taccetti, Macro and Micro Full Field X-Ray Fluorescence with an X-Ray Pinhole Camera Presenting High Energy and High Spatial Resolution, Anal. Chem. 86 (21) (2014) 10892

[17] L. Celona, G. Ciavola, F. Consoli, S. Gammino, F. Maimone, D. Mascali, P. Spädtke, K. Tinschert, R. Lang, J. Mäder, J. Roßbach, S. Barbarino and R. S. Catalano, Observations of the frequency tuning effect in the 14GHz14GHz CAPRICE ion source, Rev. Sci. Instrum. 79 (2008) 023305

[18] D. Mascali, S. Gammino, L. Celona, G. Ciavola, Towards a better comprehension of plasma formation and heating in high performance ECRIS, Rev. Sci. Instrum. 83 (2012) 02A336

[19] G. Torrisi, G. Sorbello, O. Leonardi, D. Mascali, L. Celona and S. Gammino, A new launching scheme for ECR plasma based on two-waveguides-array, Microwave and Optical Technology Letters 58:11 (2016) 2629

[20] G. Torrisi, D. Mascali, G. Sorbello, L. Neri, L. Celona, G. Castro, T. Isernia and S. Gammino, Full-wave FEM simulations of electromagnetic waves in strongly magnetized nonhomogeneous plasma, Journal of Electromagnetic Waves and Applications 28:9 (2014) 1085

[21] D. Mascali , G. Torrisi, L. Neri , G. Sorbello, G. Castro, L. Celona, and S. Gammino, 3D-full wave and kinetics Numerical modelling of Electron Cyclotron Resonance Ion Sources plasma: steps towards selfconsistency, Eur. Phys. J. D 69:27 (2015) 9pp

[22] G. Melin, C. Barué, F. Bourg, P. Briand, J. Debernardi, M. Delaunay, R. Geller, A. Girard, K. S. Golovanivsky, D. Hitz, B. Jacquot, P. Ludwig, J.m. Mathonnet, T. K. Nguyen, L. Pin, M. Pontonnier, J. C. Rocco and F. Zadworny, 1990 Proc. 10th Int. Workshop on ECRIS (Knoxville, TN, 1-2 November 1990) pp 1-15 